\title{
Maximized Congestion Control in TCP with Multi-Path Support in Wireless Cobweb Network
}

\author{
Dr. Reshma Banu ${ }^{1}$, Ayesha Taranum ${ }^{2}$ \\ Professor \& HOD ISE, GSSSIETW, Mysore ${ }^{1}$ \\ Assistant Professor, Dept of ISE, GSSSIETW, Mysore ${ }^{2}$
}

\begin{abstract}
Wireless Cobweb Network (WCN)[1] is a group of wireless devices that has function to transfer all data over wireless channel and it is able to self-organized, self-configure network path itself by optimize routing protocol over Layer 2 (MAC Layer). The WCN can be installed by using computer or device with interface base on IEEE 802.11 forming to the WCN. This network can provide services with any content as wiring network. In IEEE $802.11 \mathrm{~s}$ [2] is standard for Wireless Cobweb Network (WCN) which improve the communication protocol in Layer-2 (MAC Layer), using the Hybrid technic from the advantage of ad-hoc base and tree base for routing with path in WCN. With the wireless network standard came from IEEE $802.11 \mathrm{a} / \mathrm{b} / \mathrm{g} / \mathrm{n}$ standard which does not define to build for the WCN and is different from the wireless access network (2G/3G Cellular systems,WLAN hotspot) [1].Wireless Cobweb Network is consisted of three type of node; Cobweb Portal (CPP), Cobweb Point (CP), Cobweb Access Point (CAP) [1]. All devices came with IEEE802.11 Standard and beyond - i.e., the physical layer link base on CSMA/CA[3] and standard TCP that work well with Wire Topology but may not suitable with WCN and optimize is in required. Regarding TCP/IP Model, many researches are focused to optimize any layers from physical to application. This research would be focused on the method to optimized congestion control in TCP with Multi-Path support over wireless Cobweb network.
\end{abstract}

Keywords: TCP, Congestion control, 802.11s Wireless Cobweb network, Multi-path, Optimization.

\section{INTRODUCTION}

The IEEE 802.11 wireless standard has posted and more hardware are compiled onto many devices like computers also mobile phones do exchange and transfer data over wireless channels. The typical 802.11 network is based on a single access point (AP) which is connected to communicate to the internet over wire-line (an Ethernet Cable). In the previous to coverage these service over the area need to deploy more AP where need wire-line also. Wireless Cobweb network (WCN) provides a solution to reduce cost over wired line between APs. The IEEE 802.11s become a standard for wireless device to work over Cobweb network standard. The Cobweb link is shared by minimum two nodes. Any node has services of control, management and operation of the Cobweb is a Cobweb Point (CP). The node which connects to other networks such as internet is a Cobweb Portal (CPP). The other node is providing wireless service to client station (STAs) is called Cobweb Access Point (CAP).

All Cobweb nodes has connection link via wireless radio over IEEE $802.11 \mathrm{a} / \mathrm{b} / \mathrm{g} / \mathrm{n}(2.4 \mathrm{GHz} / 5 \mathrm{GHz})$ and later become standards. WCN nodes can support multi-interfaces radio link and multi-channel on radio interfaces. Cobweb nodes would initial a channel to perform active or passive scanning of the Cobweb nodes neighbor CPs will probe all nodes by using calculation radio air time metrics [3] to selection and routing on path to the next hop node.

The Cobweb routing using Hybrid Wireless Cobweb Protocol (HWCP) for crated route table which is over on Layer-2 by Mac Address $[2,4,5]$. The congestion event might occur in every Layer from Lower e.g. Physical, Data-Link. And the Upper layer in Transport layer e.g., Transmission Control Protocol (TCP), User Datagram Protocol (UDP) using by forward up and down to protocol stacks layer. In WCN in Layer2 protocol design use MAC address as header to send packet to the destination by hop via next MAC address of neighbor nodes. The Inter- Cobweb congestion [7] will occur for management the Cobweb operation protocols and will impact to Transport Layer. there is no scheme specified in IEEE802.11s for the local rate control and cannot achieve by EDCA[4,5,6,7] parameters which is Random Backoff Contention Window under Data Link Layer by CSMA/CA. In TCP has Congestion avoidance algorithm to determine how much bandwidth is available in the network and adjust its transmission rate accordingly. Its control strategy, referred to as Additive Increase/Multiplicative Decrease (AIMD), handle from being overloaded and stability network. In the network is not handle only TCP this will cause the congestion to control transmitting packets thought the destination via bottleneck link or was dropped because over queue buffer of router. This paper is optimized the congestion control in TCP on WCN with has Multi-Path. 


\section{LITERATURE REVIEW}

Congestion window size was defined as saw-tooth shape via packet cycle (e.g. round trip time (RTT)) from sender to receiver [8] into every segments. The steady-state model is define as $\mathrm{T}$ is a retransmission timeout.

$$
T=\frac{\text { Data per cycle }}{\text { Time per cycle }}=\frac{B-W^{2}}{R T T \frac{W}{2}}=\frac{B / p}{R T T \sqrt{\frac{2}{3 p}}}=\frac{1.5 \sqrt{2 / 3 B}}{R T T \sqrt{p}}
$$

The model of TCP congestion control behavior in steady state, which includes the effect of time out and retransmission as shown below. In this case T0 is a retransmission timeout, and Wmax is the maximum congestion windows when it is limited by the buffer size at the receiver.

$$
T \approx \min \left(\frac{W_{\max }}{R T T}, \frac{1}{R T T \sqrt{\frac{2 B p}{3}+T_{0} \min \left(1,3 \sqrt{\frac{3 B p}{8}}\right) p\left(1+32 p^{2}\right)}}\right)
$$

Varies TCP versions to optimized congestion control and window size over network test bed setup. In [10] they setup a dumbbell network to compare the performance of TCP-NewReno with other on high-speed network. They found the HTCP modified protocol has a best results. In [11] they developed TCP - friendly CBR-Link Rate Control (TFCBR) for smooth sending and not require high-speed network by estimate rate by averaged TCP-friendly rate control (TFRC) in the past second. The author [12] shown the study performance of TCP over wireless network is varies by the lower layer control the packet delay and loss affect to the TCP congestion control algorithm to retransmission the packet. TCP Vegas is a delay base TCP by adopt to estimation bandwidth and avoid congestion than react by measured RTT. The author [13] proposed a new mechanism called Competitive Delay-Based TCP (CODE TCP) which is a variant of TCP Vegas.

\section{A. TCP Reno}

- The congestion control is start by slow-start state by set congestion windows (cWnd) size equal segment size base on discovered MTU size at initial. The cWnd will Additive Increase/Multiplicative Decrease (AIMD) by every NewAck from the sequence packet by $\mathrm{cWnd}=(\mathrm{cWnd}+$ Segment Size $)$ until not over than Threshold size is 65535 Bytes. When retransmission state occur because packet was dropped or loss. The Threshold was reduce to half of flight size and cWnd is set to segment size. When Threshold size changed by retransmission timeout (RTO). TCP Reno would enter in congestion avoid mode by increase cWnd used (segment size $\mathrm{x}$ segment size) / cWnd size. When Triple DupAck occur $\mathrm{cWnd}$ is set size to (Threshold +3 Times of segment size) and set in fast recovery state is true. The other DupAck if the fast recovery is true the $\mathrm{cWnd}=\mathrm{cWnd}+$ segment size.

\section{B. TCP NewReno}

- The NewAck state is enhanced than TCP Reno. All slow-start, congestion avoidance, retransmit are same as Reno. When NewAck arrived after got Triple DupAck, The protocol would fill the last sent sequence packet and change the Fast Recovery state, if current seq number is less than last seq number then new $\mathrm{cWnd}=(\mathrm{cWnd}-\mathrm{seq}$ Lastseq) and increase $\mathrm{cWnd}=\mathrm{cWnd}+$ segment size. Otherwise, $\mathrm{cWnd}$ would increase as last Byte was sent and plus with segment size.

\section{TCP Tahoe}

- $\quad$ The TCP Tahoe has slow-start and congestion avoidance as Reno but does not have the Fast Recovery State after got Triple DupAck. Threshold size was reduced to half of last $\mathrm{cWnd}$ and $\mathrm{cWnd}$ back to initial size even into retransmit.

\section{TCP Vegas}

- This protocol [10] adopts more than other TCP congestion avoidance technic by using estimation of bandwidth measured from RTT to calculate how source can send

\section{E. TCP Westwood}

- The basic of TCP Westwood protocol is to estimate the current bandwidth. At slow-start and congestion avoidance is similar as Reno. This will calculate by estimate bandwidth on ReceivedAck which is a duplicate ACK or a new ACK and set Threshold equal current bandwidth by estimation from counting TCP header ACK * segment size / ACK Time. If cWnd is larger than Threshold size after DupACK. It will reset to equal Threshold size. 
The TCP protocol was designed to optimize the specific problem such as on mobility management where networks using a handover from station to another station by radio link. The author $[14,15,17]$ shown varies different end-to-end protocols e.g. TCP-R, TCP-Migrate, pTCP, obTCP, etc. In [16] the framework of Markov Decision Process is using to optimal queue management in a bottleneck router to fast and fair transmission. The TCP-Friendly Rate Control (TFRC) is popular rate control over wired network. It assumes perfect link quality and treating network congestion as packet losses. The author [18] proposed a cross-layer optimized end-to-end TFRC for real-time multimedia over wireless multi-hop network. In [19] they proposed IFTP protocol is internet friendly transmission protocol for multimedia applications. Because they found TCP was not designed with wireless problem it's interpret the cause of packet drop as indicated by either retransmission timer expiration or the receipt of multiple duplicate ACKs as network congestion. The IFTP is enhanced from TCP-Reno by add the calculation of RTTbase and RTTactual.

The author in [20] enhance by cross layer for end-to-end throughput by using lower layer. The proposal is assigned Cobweb node has multi-channel. The Cobweb nodes has inter-node link is directed link between nodes. They define intra-node links on nodes another interface as multi-links. The Cobweb node in IEEE802.11s using air time cost to determine which link is operate to the next-hop nodes. the author in [21,22] proposed the Cross-Layer Service Middleware(CLSM) designed to monitor the airtime cost calculation and link to Listening socket to comprehensive metrics (e.g. frame error rate,TCP buffer size and timestamp support) according to the request of applications. We will found that most of TCP end-to-end connection has link over an interface through other networks. the author in [23] using Multipath TCP protocol (MPTCP) is a complicated transport layer protocol that transfers data by multiple paths simultaneously and proposed Path Associativity Congestion Control (PACC). These control the sub-flow which has congestion control by count bytes_Acked to the cWnd increment in slow-start phase. The author in [24] proposed minimum queue length load-balancing (MQLLB) is dynamic algorithm to manage multi-path forwarding on node topology as figure [fig.1 and fig.2].

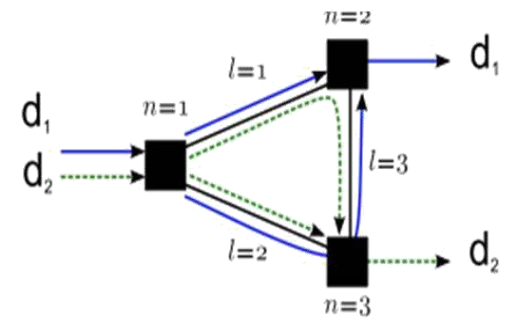

Fig. 1. 3-Node topology multipath forwarding example

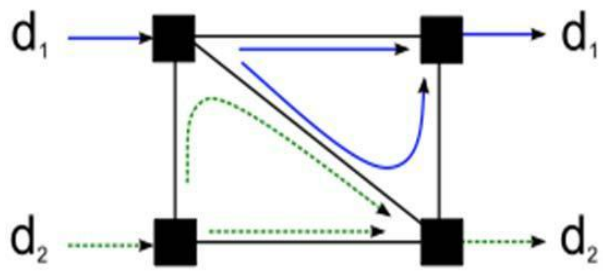

Fig. 2. 3-Node topology multipath forwarding example

\section{METHODS}

From the previous section, we found the problem when TCP protocol was not optimize over WCN or multi-hop networks, the congestion control need to develop to control the cWnd packet sizes over unstable network as wireless signature. The packet could loss or delay by lower layer control protocols or an interference affect the radio link. We propose an optimize congestion control by design cross-layer of TCP protocol. The Markov Chain steady transition state calculation for measure cWnd sizes in Slow-Start and congestion avoidance state. The cross-layer will applied the Cobweb nodes to send the packets over multi-path by measure an available bandwidth on each interface and each path. It will share packet send and receive load on next-hop Cobweb nodes links.

We modified Cobweb L2HWCPProtocol in NS3 and customize the TCP Congestion windows size. In Cobweb L2HWCP protocol after got packet from neighbor node and defined to forward to other node by finding match destination MAC address in routing table. We found that if it cannot found the MAC address following the HWCP Protocol it would return the MAC address of neighbor node where path to the Root Node. I assume that the another Cobweb Link path should consider to try send packet to the lowest metric to reach a packet to the destination. We also modified the congestion window size based on TCP Reno by calculate from RTT and Bandwidth Delay Product (BDP) of Cobweb Network. The window size estimate by below We setup Cobweb network on NS3 simulation. The Cobweb topology similar and dumbbell with Cobweb network between and Station (STA) is shown in Fig.4. The Cobweb node 
is set Data Rate at 5Mbps. The 802.11s PeerManagementProtocol is enable to avoid the BeaconCollision. The Physical is set to 802.11a and Cobweb nodes had only one interface. The STA is serial-link devices attach to the Cobweb Node as CPP.

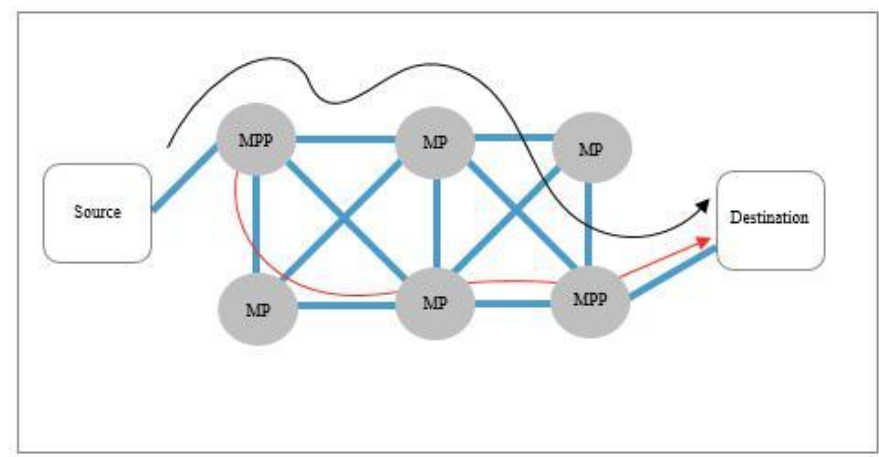

Fig. 3. Optimized Cobweb L2HWCP Protocol to find the lowest metric route

We test by sent packet size 2,500 bytes with 10,000 packets. We measured the optimized TCP by below

$$
\text { PDF }=\frac{\text { Total Rx Packet }}{\text { Total Tx Packet }} \%
$$

\section{IV.CONCLUSIONS}

This paper proposed an optimized congestion control on Wireless Cobweb Network. RTT and BDP is used for calculating a best window size for on an environment of wireless radio links. Because, Standard TCP Protocols was design on the stable link and does not define the pattern of packet lost might occur often than wired link. The hop count of the radio link is a bottle neck of bandwidth. The result of simulation on NS3 shown our congestion calculate size while transfer packet over WCN. The pattern of congestion window size and number of packet loss of our modified TCP could transfer data better than other. The future work, we will modified an optimized TCP base on Multiple Channel.

\section{REFERENCES}

[1] 487Sandip Chakraborty, Pravati Swain, Sukumar Nandi, "Proportional fairness in MAC layer channel access of IEEE 802.11s EDCA based wireless Cobweb networks", Ad Hoc Networks 11 (2013) 570-584

[2] Germán Capehourat, Federico Larroca, Pablo Belzarena, "Optimal multipath forwarding in planned Wireless Cobweb Networks", COMCOM 4858,No. of Pages 14, Model 5G,2013

[3] Rosario G. Garroppo, Stefano Giordano, Luca Tavanti, "Implementation frameworks for IEEE 802.11s systems", Computer Communications 33 (2010) 336-349

[4] Joseph D. Camp and Edward W. Knightly, "The IEEE 802.11s Extended Service Set Cobweb Networking Standard",

[5] Xudong Wang, Azman O. Lim "IEEE 802.11s wireless Cobweb networks: Framework and challenges",Ad Hoc Networks 6 (2008) 970-984

[6] Ricardo C. Carrano, Luiz C. S. Magalhães, Débora C. Muchaluat Saade and Célio V. N. Albuquerque, "IEEE 802.11s Multihop MAC: A Tutorial", IEEE COMMUNICATIONS SURVEYS \& TUTORIALS, VOL. 13, NO. 1, FIRST QUARTER 2011

[7] Salim Nahle and Naceur Malouch, "Graph-based Approach for Enhancing Capacity and Fairness in Wireless Cobweb Networks",IEEE "GLOBECOM" 2009

[8] Gwyn Chatranon, Miguel A. Labrador, Sujata Banerjee, "A survey of TCP-friendly router-based AQM schemes", Computer Communications 27 (2004) 1424-1440

[9] Barbara Staehle,Michael Bahr,Desheng Funder Steele, "Intra- Cobweb Congestion Control for IEEE 802.11s Wireless Cobweb Networks",

[10] Sangtae Ha, Long Le, Injong Rhee, Lisong Xu, "Impact of background traffic on performance of high-speed TCP variant protocols", Computer Networks 51 (2007) 1748-1762

[11] Jie Feng, Lisong Xu, "Stochastic TCP friendliness: Expanding the design space of TCP-friendly traffic control protocols", Computer Networks 56 (2012) 745-761

[12] K. Avrachenkov, U. Ayesta, J. Doncel, P. Jacko“Congestion control of TCP flows in Internet routers by means of index policy”, Computer Networks 57 (2013) 3463-3478

[13] Bhaskar Sardar, Debashis Saha, Mahbub Hassan, "A novel enhancement of TCP for on-board IP networks with wireless cellular connectivity", Network and Computer Applications (2013),

[14] Haiyan Luo, Song Ci, Dalei Wu, Hui Tang, "End-to-end optimized TCP-friendly rate control for real-time video streaming over wireless multihop networks" , J. Vis. Commun. Image R. 21 (2010) 98-106

[15] Hala ElAarag, Andrew Moedinger, Chris Hogg, "TCP friendly protocols for media streams over heterogeneous wired-wireless networks", Computer Communications 31 (2008) 2242-2256

[16] Jian Tang, Guoliang Xue, "Cross-Layer Design for End-to-End Throughput and Fairness Enhancement in Multi-Channel Wireless Cobweb. 